\title{
Considering Agency and Data Granularity in the Design of Visualization Tools
}

\author{
Gonzalo Gabriel Méndez ${ }^{1,2}$ \\ ${ }^{1}$ SACHI, University of St Andrews, UK \\ \{ggm, mans, uh3\}@st-andrews.ac.uk
}

\author{
Miguel A. Nacenta ${ }^{1} \quad$ Uta Hinrichs $^{1}$ \\ ${ }^{2}$ Escuela Superior Politécnica del Litoral, Ecuador \\ gmendez@espol.edu.ec
}

\begin{abstract}
Previous research has identified trade-offs when it comes to designing visualization tools. While constructive "bottomup" tools promote a hands-on, user-driven design process that enables a deep understanding and control of the visual mapping, automated tools are more efficient and allow people to rapidly explore complex alternative designs, often at the cost of transparency. We investigate how to design visualization tools that support a user-driven, transparent design process while enabling efficiency and automation, through a series of design workshops that looked at how both visualization experts and novices approach this problem. Participants produced a variety of solutions that range from example-based approaches expanding constructive visualization to solutions in which the visualization tool infers solutions on behalf of the designer, e.g., based on data attributes. On a higher level, these findings highlight agency and granularity as dimensions that can guide the design of visualization tools in this space.
\end{abstract}

\section{Author Keywords}

Visualization; Constructive Visualization; iVoLVER; Agency; Granularity; Tool Design; Tableau.

\section{ACM Classification Keywords}

H.5.m Information Interfaces and Presentation (e.g. HCI): Miscellaneous; D.1.7 Software: Visual Programming

\section{INTRODUCTION}

An increasing number of tools support visualization construction without requiring programming skills or design expertise from the users' end. This enables a larger proportion of the population to take advantage of visualization, from students and other learners, to researchers who are non-experts in visualization. Many modern commercial visualization tools, such as Tableau or MS Excel, allow the rapid creation of sophisticated visualizations and, sometimes, encourage better visualization choices. For example, Tableau makes suggestions of specific visualization types based on the selected data attributes (e.g., a bar chart, which is known to be perceptually

\footnotetext{
Permission to make digital or hard copies of all or part of this work for personal or classroom use is granted without fee provided that copies are not made or distributed for profit or commercial advantage and that copies bear this notice and the full citation on the first page. Copyrights for components of this work owned by others than the author(s) must be honored. Abstracting with credit is permitted. To copy otherwise, or republish, to post on servers or to redistribute to lists, requires prior specific permission and/or a fee. Request permissions from permissions@ acm.org.

CHI 2018, April 21-26, 2018, Montreal, QC, Canada

(C) 2018 Copyright held by the owner/author(s). Publication rights licensed to ACM. ISBN 978-1-4503-5620-6/18/04 . .\$15.00

DOI: https://doi.org/10.1145/3173574.3174212
}

efficient for displaying groups of scale quantities). As visualization tools become more sophisticated, they tend to make more choices and actions on behalf of the designer. In some extremes the tool can generate a visualization from provided data with minimal or no human intervention [25].

Somewhat in opposition to these tools stands the subarea of constructive visualization $[28,30,32]$, which is based on educational theories $[1,51,52]$. Constructive visualization promotes a more hands-on, even manual approach to visualization for a better understanding of its principles and values. Some existing evidence shows that requiring more effort from the designer has advantages in terms of learning and understanding [30], as well as an increased feeling of authorship and transparency [44]. However, the fundamental limitation of these constructive tools (e.g., [45], and their physical counterparts $[6,29,32])$ is that they do not scale to larger datasets and can be tedious and repetitive to use [44].

In this paper we investigate how to reconcile automation and computer-assisted design with the benefits of constructive visualization. To gain an understanding of this design space we conducted an iterative in-house design process as well as a series of design workshops with people of different backgrounds and expertise levels. Based on our detailed analysis of resulting solutions, also in the light of existing tools, we describe possible ways to design visualization tools that retain the advantages of both worlds. Our paper contributes: (1) a conceptualization of the problem of designing more automated constructive visualization tools, (2) a catalog of categorized design examples, (3) a characterization of this design space in terms of agency and granularity, and (4) a discussion on the implications for designing better visualization tools.

\section{RELATED WORK}

Our research draws on previous design approaches to visualization tools, including constructive visualization, and the concepts of automation, agency, and abstraction in relation to designing visualization tools as described below.

\section{Visualization Tools: Types and Approaches}

Visualization tools can be categorized in different ways [39, $43,50,67]$. For example, Grammel et al. [19] classify tools based on their user interface, labeling Tableau [62] as a "shelf configuration" tool in reference to how it presents and enables interaction with visual properties and data attributes. Other tools provide visual programming environments in which designers visualize data by manipulating graphical objects. DataMeadow [14], ExPlates [34], and iVoLVER [45] are examples of this group. In these tools the visualization process 
takes place within a canvas where graphical elements of different types process and exchange data through connections. Such tools do not require textual programming skills as opposed to specialized languages and libraries (e.g., D3 [7]).

Méndez et al. [44] discuss approaches supported by visualization tools as a continuum between two ends: bottomup and top-down. The former, represented by tools such as iVoLVER [45], promote a hands-on, constructive visualization process [28, 30,32], but are limited in terms of scalability. The latter (e.g., Tableau) allow rapid design at the cost of transparency. Aiming to marry the benefits of both approaches, we investigate design strategies that support the visualization of large datasets while maintaining a constructive, transparent, and largely user-controlled visualization process.

\section{Automation and Agency}

In visualization tools, automation facilitates visual mapping of large datasets enabling designers to rapidly explore different visual representations [44]. Many existing tools feature recommendation modules that suggest designs as users manipulate data elements (e.g., Tableau's "Show Me" [40] and similar strategies in other tools $[8,18,56,58,72])$. In general, automation is meant to ease tasks that would be otherwise unnecessarily difficult, repetitive, or tedious. However, research from psychology and cognitive sciences has shown that too much automation may lead to complacency and bias [54, 53]. Relying on the computer, people are less likely to reflect on how automated routines act on their behalf [13]. Furthermore, people often give more weight to the computer's decisions even in the presence of contradicting evidence [10].

In digital tools, excessive automation can also affect how people perceive agency. Agency “refers to a person's ability to control their actions and, through them, events in the external world." [20, p. 242]. The experience of agency is an important aspect of HCI research because users "strongly desire the sense that they are in charge of the system and that the system responds to their actions." [59, p. 75]. As shown by Coyle et al. [12], too much computer assistance can harm people's sense of agency in a point-and-click task.

The InfoVis community has also paid attention to agency. People experience different levels of control and authorship of the visualization design process when working with tools that implement opposite levels of automation [44]. Tori and Möller's taxonomy emphasizes the human aspect of visualization techniques by considering "how much the designer chooses display attributes" [69, p. 154]. Koytek et al. showed that incorporating personal agency into brushing and linking interaction [37] can increase the transparency of a visualization tool. To better preserve people's sense of agency while exploiting computational power, other works combine automation and human involvement (e.g., $[4,47,55])$. Along these lines, Yu and Blackwell investigated the role of timing on people's perception of agency in mixed-initiative interaction [73].

\section{Abstraction}

To cope with datasets of different types and scales, visualization tools often create abstractions of the data and visual mapping processes. While constructive visualization approaches have a low level of abstraction (as values are directly mapped to visual elements), most commercial tools implement higher levels of abstraction on a data, visual, or interaction level. Abstraction is a cognitive process that plays an important role in human language and thought [24, p. 184]. It is a complex term discussed across many areas of study such as philosophy [57], psychology $[16,60]$ and mathematics [21]. It is used in engineering to cope with complexity [36], although it necessarily implies hiding of detail. In the remainder of this paper we will refer to one specific type of abstraction-generalization abstraction-which we define narrowly as the ability to refer to a set of items with a symbol. For example, when dealing with data, the name of a column becomes an abstraction of all the values contained in that column. Abstraction can also be recursive: we can abstract a group of abstractions (e.g., referring to the Name, Surname, and Age columns as the Person data table increases abstraction). Our definition of abstraction is related to encapsulation in Software Engineering and Object-oriented Programming [61], and it has been discussed in the context of visualizing algorithms [46].

\section{GOALS AND METHODOLOGY}

Our research aims at bringing together the benefits of bottomup and top-down approaches to visualization tools. This is a difficult challenge as the advantages of bottom-up visualization tools - a deep involvement and understanding of the visualization process - seem to be incompatible with the benefits of top-down visualization tools: the quick and easy visualization, even of large datasets, made possible through automation [44]. To investigate possible solutions to this challenge in-depth and from multiple perspectives, we followed a mixed design methodology driven by: (1) our own expertise as visualization researchers with significant experience in designing visualization tools and UIs, and (2) the ideas from a larger group of people with varying degrees of expertise in visualization and visualization tools, who took part in four design workshops we organized. In the search of design approaches to visualization tools that would be suitable not only to experts but also to visualization novices, we found the involvement of a larger group of people with different backgrounds to be important. The constructive visualization tool iVoLVER [45] provided a starting point for our explorations, which focused on revising its design to make it suitable for visualizing larger datasets while maintaining transparency and user-involvement in the visualization process. In the following we first discuss the in-house design solutions that we came up with as experts in visualization tool design. This is followed by a discussion of the design workshops and the solutions proposed by the workshop participants.

\section{IN-HOUSE DESIGN SOLUTIONS}

To explore solutions to the problem of visualizing large-scale datasets in the constructive tool iVoLVER, two of the authors of this work engaged in an iterative design process. We aimed at finding a "sweet spot" between the two ends of the bottomup vs. top-down continuum [44], balancing their trade-offs from an expert perspective. This process consisted of four meetings of about 1.5 hours each in which we developed and incrementally refined a large number of initial paper sketches At the end we consolidated the designs into three representative in-house solutions: 


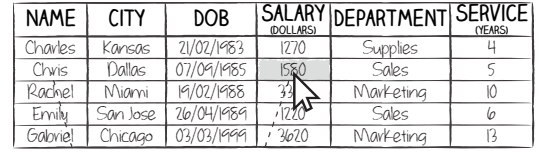

(3) W'E CONNECT VALUES FROM THE' TABLE TO THE

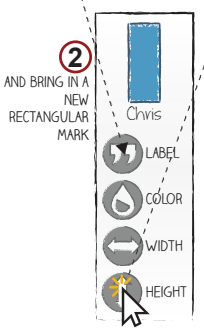

(1) Weactivate the ReCording

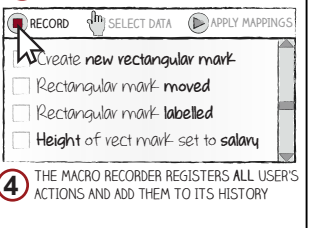

(4) ACTIONS AND ADD THEM TO ITS HISTORY

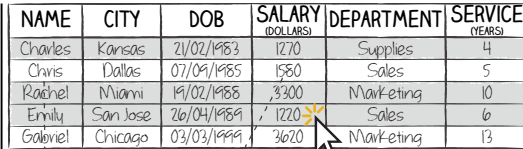

(6) WE SELECT DATA RECORDS BY

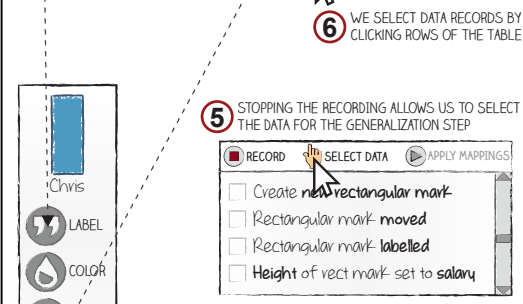

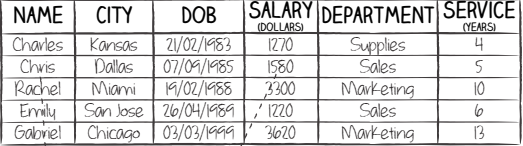

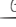

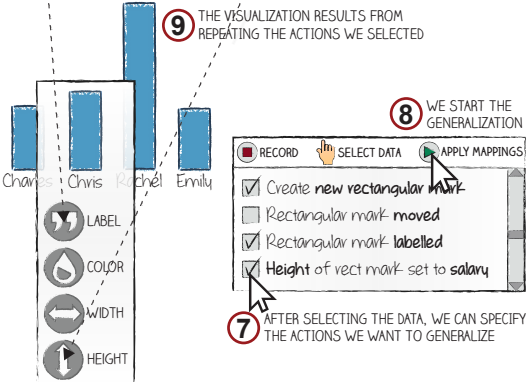

Figure 1: In-house Macro Recorder.

Macro Recorder: In this solution, users specify visual mappings by connecting individual values of the dataset (i.e., cells of the data table) to a mark's visual properties while an additional interface element, the Macro Recorder, registers the history of manual actions throughout the visualization process (Fig. 1, left). The recording is activated upon request. When finished it allows the selection of (1) a subset of the performed actions and (2) a set of data (i.e., rows of the dataset) to which the selected actions will be applied (Fig. 1, middle). This generalization step results in a set of new marks that represent the selected data according to the mappings specified by the actions on the initial mark (Fig. 1, right).

This design is based on the concept of demonstrational interfaces that "let the user perform actions on concrete example objects [...] while constructing an abstract program" [48, p. 61]. It preserves iVoLVER's bottom-up approach as it allows people to drive the visualization design process by specifying mappings at the level of individual data values and marks. The recording feature allows for generalization and, in this way, resolves the problem of having to repeat individual interaction steps, one of iVoLVER's main drawbacks [44, 45].

Smart Assistant: This solution proposes a visualization process based on a mixed-initiative interaction approach [2]. Visual mappings are specified based on a single mark and data value. The system monitors the designer's mapping actions and provides guidance on how to proceed in further stages of the visualization construction process. As this Smart Assistant detects a new mark or modification of a visual property, it infers how the mapping applies to other data and shows previews of the corresponding visualization based on all data values (Fig. 2, left). The user can accept or reject these suggestions, or explore alternatives (Fig. 2, middle \& right).

Proxy Mark Widget: In this solution mappings are specified not directly on marks, but through a widget which acts as a proxy for a group of marks and provides access to their visual properties. Users specify visual mappings by feeding sets of data values to the widget's graphical representations of visual properties (see Fig. 3.1). These sets can contain either all the values associated to a data attribute or just some parts. In our design, the former are available through the column headers of the data table while the latter can be manually constructed with a vertical dragging gesture that spans across the desired values. Once all the data mappings have been specified, an output port in the widget allows users to drag the resulting visualization to the canvas (see Fig. $3.2 \& 3.3$ ).

This solution is similar to Tableau's "Marks Card" [65], with the important difference that the user is in full control of the visual properties of marks, including their position on the canvas which can be defined explicitly, rather than implicitly as in Tableau through the "Columns and Rows" shelves [63].

\section{DESIGN WORKSHOPS}

We conducted four design workshops to expand and validate our ideas on how to facilitate the visualization of large-scale datasets in iVoLVER. We deliberately chose participants from a range of backgrounds and expertise to engage with the problem for half a day in groups of 3-4. Since the expert perspective is already represented (see above), we wanted to encourage solutions from other fields, beyond experts' perspectives on current tools and beyond the state of the art. To provide participants with some background and to expose them to contrasting visualization authoring approaches, they first worked with Tableau and iVoLVER (two extremes in the space between top-down and bottom-up visualization approaches [44]), before they sketched and discussed their own design ideas.

\section{Participants}

We recruited 14 participants from a local university ( 8 female, 19-49 years old; median 26) ; 12 were undergraduate or graduate students in computer science, mathematics, English literature, or museum studies; one was a research communication practitioner, and one a lecturer in economics.

Expertise in visualization varied from no knowledge $(n=1)$, web or print from a consumer perspective $(n=7)$, awareness of concepts without practical experience $(\mathrm{n}=3)$, and visualization practitioners with at least one year experience $(n=3)$.

Participants' experience with visualization tools and interface design varied. Six did not know about Tableau; three had heard of it. Four had used it once, and one was comfortable with it but not proficient. Five participants did not know iVoLVER; five had heard of it. One had used it once, and three were comfortable with it, but not proficient. Four participants had no experience with designing UIs. Nine had varied levels of expertise, ranging from novices $(\mathrm{n}=1)$, to basic knowledge from university projects $(n=4)$, to practitioners with at least 

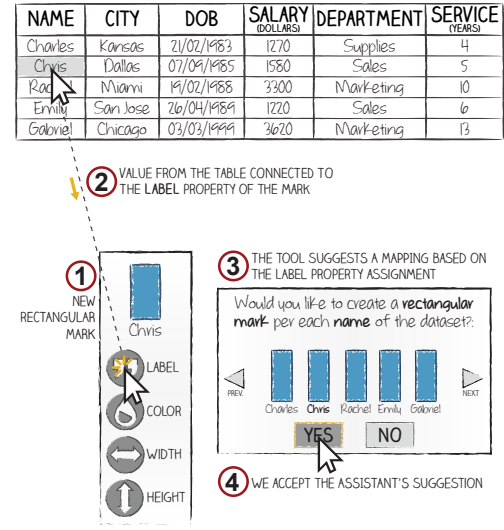
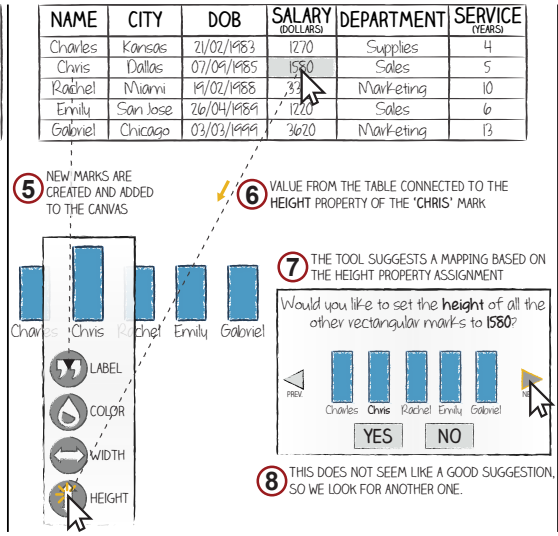

Figure 2: In-house Smart Assistant.

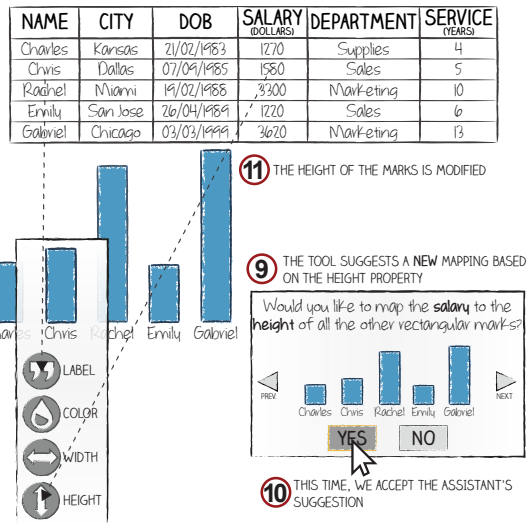

Each dataset (one about oil production, one about movies, both used also in [44]) consisted of five data points and five attributes. The tasks included the creation of three different visualizations involving different data attributes. The tasks for both datasets were equivalent in the type and number of involved attributes. Participants had 30 minutes to complete the tasks using a laptop computer with their preferred input method (e.g., mouse, touchpad). After this, they were introduced to the second tool and completed the visualization tasks using this tool with the second dataset.

Group Discussion on Tools. The workshop attendees briefly discussed their overall experience with Tableau and iVoLVER, including differences, advantages and limitations.

Design Briefing. These hands-on visualization sessions were followed by a briefing highlighting the main goals for the sketching session: To revise iVoLVER as a constructive visualization tool that would (1) scale to large datasets, (2) support a speedy visualization process, and (3) avoid repetitive interaction steps; all while still supporting transparency and keeping the user in charge of the design decisions.

Sketching Session. Participants then spent approx. 45 minutes sketching design solutions that would meet these goals. They were advised to revising iVoLVER by either modifying its current features or creating new ones. Participants were provided a sketching toolkit that, among other materials, contained several printed cutouts of a sample dataset (different from the data used in the visualization exercise) that they could glue to A3 sketching sheets as a starting point for sketching a new idea and as a common ground to discuss all participants' solutions later in the workshop. The dataset representations came in small (five records/rows) and large (36 records) versions.

Presentation of Sketches. Participants presented their sketched solutions to the group, explaining their ideas toward a better constructive visualization tool.

Participants did not collaborate at any point during the workshop session. The workshops ended with us presenting our in-house design solutions leading into a concluding discussion of the ideas that had come up in the workshop.

Data Collection \& Analysis

Participants' presentations of sketches were video-recorded (98 min. in total), and we collected all produced sketches 


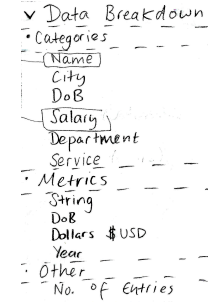

(a) Displaying [P8].

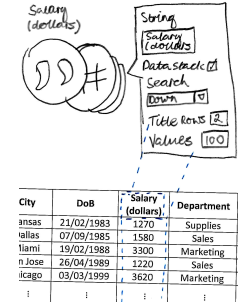

(b) Formatting [P4].

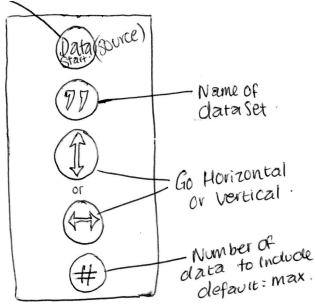

(c) Formatting [P5].

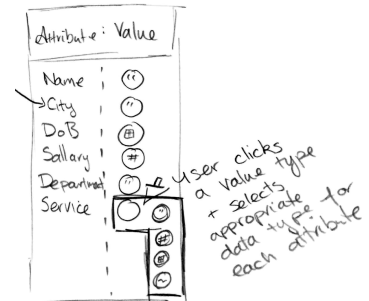

(d) Setting the data type [P2].

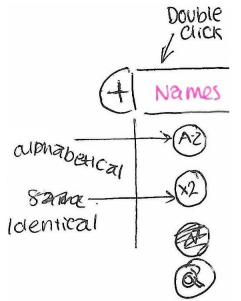

(e) Sorting/filtering [P5].

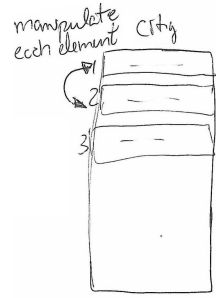

(f) Rearranging [P3]

Figure 4: Designs that address data-related aspects of the visualization construction process .

(41 pages). This data forms the basis for our findings. One of the authors of this work (the facilitator of the workshops) analyzed the video data following a video coding process as proposed by Heath et al. [22]. Observational notes taken throughout the workshops informed this process. Initial coding focused on the visualization stages as addressed by participants and how they envisioned particular features and their implementation. This was followed by grouping these initial codes to classify the different design solutions based on similarities and higher-level design strategies. All three authors participated in this grouping of codes.

\section{RESULTS}

Participants' approaches to the introductory visualization tasks confirm observations of previous research that compared bottom-up versus top-down visualization authoring using iVoLVER and Tableau [44]: participants explored more alternative designs when working with Tableau, and produced a more varied set of visualizations when working in iVoLVER ${ }^{2}$. Along the same lines, the group discussions focused on the trade-offs of the two tools' approaches, namely the speed of construction and automation (Tableau) and the transparency and flexibility of the visualization process (iVoLVER). This validates the setup of the workshops and the understanding of the problem in preparation for the sketching session.

Categorizing the participants' sketches, we found that these address three aspects of the visualization process as described below: (1) data preparation (e.g., formatting, sorting, filtering), (2) interactions with the data (e.g., grouping, creating data subsets), and (3) visual mappings specification. Note that some sketches are annotated in yellow for better comprehension.

\section{Data Preparation Support}

Six sketches (all produced by participants with a computer science background) included solutions to pre-process the data before starting the actual visualization process. Participants with non-technical backgrounds simply assumed that the tool would recognize the data's structure automatically.

Most of these designs extract and format the data from the table to reflect the relationships between values and attributes. For example, P8 proposed an automatic approach where the tool generates a "data breakdown" pane which organizes the data according to attribute types (see Fig. 4a), resembling Tableau's Data Pane [64] in appearance and functionality. Other participants proposed a more manual approach for data configuration and extraction. For example, P4 designed a widget to extract

\footnotetext{
${ }^{2}$ The produced visualizations and complete set of design sketches are available at http://ivolver.cs.st-andrews.ac.uk/scalability
}

and format the values from an attribute's column (Fig. 4b) which also allows other specifications (e.g., the number of values to extract). P5 proposed a similar element that extracts and formats values also across columns (Fig. 4c), P2 designed a widget to set the data type of each attribute (Fig. 4d). Other participants designed configurable canvas elements to filter and sort data (Fig. 4e) and to rearrange the individual values of an attribute (Fig. 4f).

\section{Interactions with the Data}

Although some design ideas required interaction with individual data values, most participants proposed manipulating several values at a time, highlighting that working with individual values makes the process tedious: "Both of my ideas are targeted towards improving efficiency, because I found that [in iVoLVER] some of the tasks were quite tedious. Especially since it's all geared towards single values." [P8].

11/14 participants proposed accessing all values of an attribute by interacting with the column headers of the data table (Fig. 5a): "We want to go from creating data bindings between a single value to some property of an object [a mark]. And we want to go from that to potentially manipulating thousands of objects [marks] at the same time. So, the natural thing to do is to consider the headings, the column headings for the data that we have." [P7]. The creation of value subsets of an attribute was commonly supported, for example, via dragging gestures to select the values of interest (Fig 5b). Similar to the selection of nonadjacent cells in MS Excel, P13 proposed to "select multiple parts of your data [...] via drag-and-drop or "control plus click'”.

\section{Specifying Visual Mappings}

17 design ideas presented by participants focused on how to specify mappings between data and visual properties. We categorized these into eight groups representing unique ideas.

\section{Mapping Replication}

P6 proposed an idea that directly builds on iVoLVER's standard way of mapping data to visuals, but replicates this map-

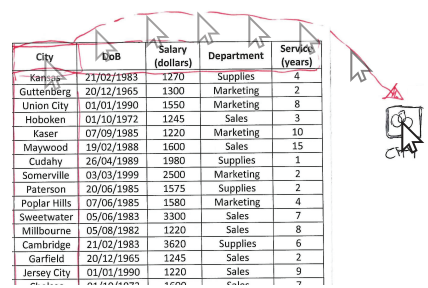

(a) With column headers [P3].

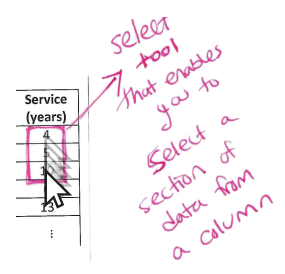

(b) Direct selection [P12].
Figure 5: Interactions with data table (shown with cursor annotation). 
ping process to multiple values and/or attributes. As shown in Figure 6a, a data value Charles has been dragged into the label property of a circular mark, creating a visible link (the visual properties of the mark are not displayed in the sketch). The same is done to a quantitative value of the Salary attribute, linked to the mark's radius. Finally, a value of Supplies is converted to color through an additional widget before being mapped to the mark's color property. Now that the first visual mapping step is completed, the user can press the "plus" popup button to replicate the same process using other data records by selecting rows in the table (Fig. 6b).

\section{Macro Recorder}

P13's idea was to "record macros to automate actions" as described in his presentation: "You would get one mark and say 'I want to take the country's name for the label, and I want to take the height of the bar-if it's a rectangle-for the population of that country'. And you would kind of record your actions. Not as literal 'go to this part of the screen with the mouse cursor and click here', but just to say 'now rinse, repeat, wax on, wax off' all the way down the table, reapplying the same set of actions as I have."

\section{Propagation of Individual Mapping Steps}

P10's approach (verbally presented) allows users to connect individual data values to visual properties and propagate the underlying mapping to other existing marks on the canvas via a gesture that takes into account the values' and marks' spatial arrangement in the data table and canvas. For example, after connecting a Salary value $V$ to the height of a rectangular mark $M$, swiping right on the canvas would modify the height of all marks located to the right of $M$ with values from Salary that are located below $V$ in the data table. Swiping left would apply the values above $V$ to the marks to the left of $M$.

\section{Bulk Modification of Mark Properties}

P2 proposed a selection tool to group marks in the visualization canvas: Figure 7a shows a sketch where four marks (highlighted in green) have been grouped. Users can access the visual properties of a mark and connect individual data values to it, which directly affects the other marks: "If you change [a visual property] for one thing [mark] in that group, that [property] changes in the same way for all of them." [P2]. This operation can make use of actual data, but it can also facilitate changing a particular visual property across all marks: "It's not intended for large datasets where the attributes need to be different for every one. It's intended for, like, if you wanna change the color of several marks at the same time." [P2].

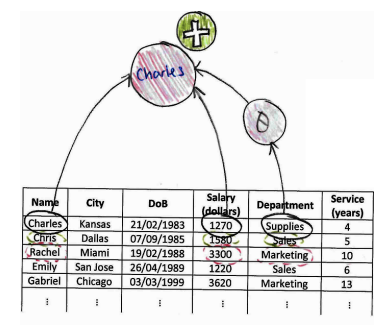

(a) Setting properties of a mark.

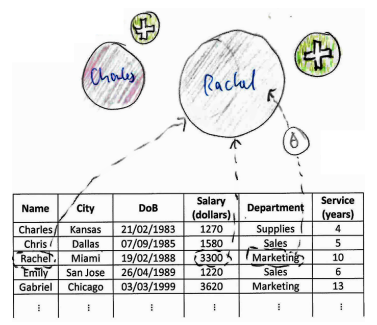

(b) Replicated mappings.
Figure 6: The plus popup button [P6].
P11 proposed an idea that also involves a group selection tool (Fig. 7b). Here, the tool automatically associates a widget to any user-created group of marks. Double-clicking a group evokes this widget which provides access to the marks' visual properties. Sets of data values can be connected to each of these properties. When receiving a new data link, the corresponding property of each mark within a group changes.

\section{Collective Proxy Objects}

Five participants (P2, 5, 6, 7, and 12) proposed specifying the mappings of several marks using a single graphical object (Fig. 8). The circular object in Figure 8a, for example, is not a mark, but a widget that provides a list of four visual properties. The sketch shows the data attributes Name, Salary, and Service connected to the widget's label, height and width visual properties. The widget eventually generates the visualization by instantiating a group of marks representing the specified mappings. We refer to these designs as collective proxy objects as they support a visualization process that involves an object that acts as a proxy for a collection of marks. Participants' ideas differed in the following aspects:

a) Shape Property. Three participants sketched ideas where the visual appearance of the proxy object determines the shape of the marks it generates. For example, a rectangular proxy object creates a bar chart like visualization (e.g., Fig. 8b). P2 and P6's ideas (Fig. 8a and 8c) are also based on a predefined shape property. To overcome the inherent limitation of this solution that only allows the construction of visualizations whose marks have the same shape, $\mathrm{P} 7$ proposed a shapeless proxy object with an additional shape property to be specified for each mark independently. P12 proposed a similar idea (see Fig. 8e; here, this property is called "mark").

b) Time \& Space of Rendering. Four proposed ideas require the user to explicitly trigger the generation of the visualization once the visual mappings are configured. For example, in P2's design (Fig. 8a), this is done via the button located at the bottom-right of the circular proxy object. Other participants proposed using drag-and-drop gestures from the proxy object onto the canvas. Only one participant proposed a design that does not require a rendering step but allows changes on marks to take place in real time, as data is connected to the proxy object's visual properties. As shown in Figure 8d (left) marks (the three black rectangles) reside within the proxy object itself, which also acts as a container for the resulting visualization.

c) Position Mappings. The proxy objects of four participants lack location properties; they implement position mappings as in iVoLVER: by linking marks to an external positioner

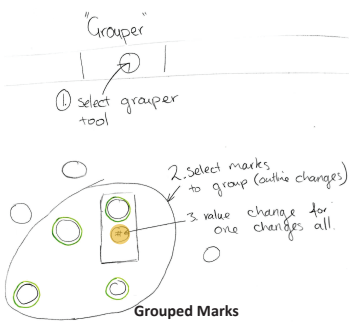

(a) P2.

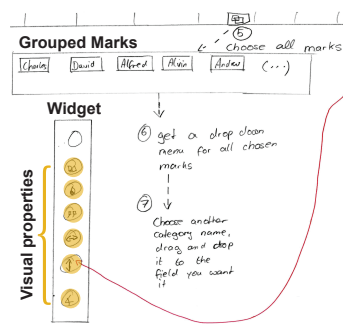

(b) P11.
Figure 7: Bulk assignation of grouped marks' properties. 


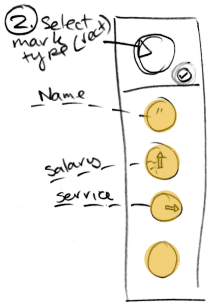

(a) P2.

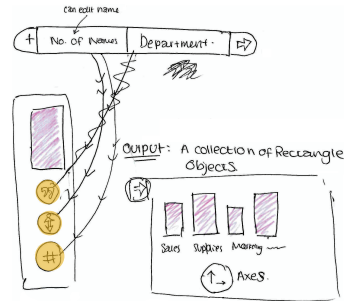

(b) P5.

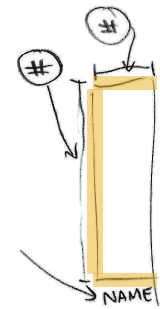

(c) P6.

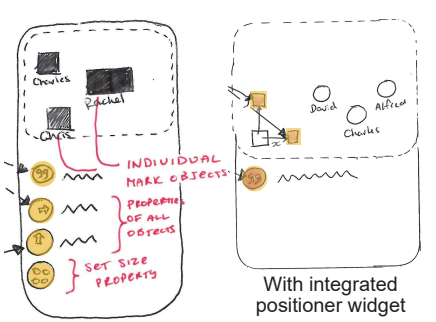

(d) P7.

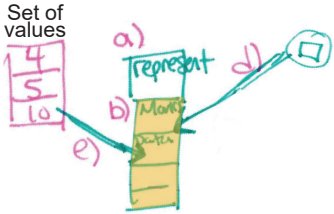

(e) P12.

Figure 8: Designs that specify visual mappings through a proxy object. The yellow highlighting shows the proxy's properties where data is connected.

widget. In contrast, P7 incorporated the positioner widget within his proxy object design (Fig. 8d, right).

\section{Axis-based Solutions}

Three participants proposed widgets that include axes as a fundamental component to enable the specification of position mappings when sets of quantitative values are connected to the widget's axes (Fig. 9). When the connected data contains categorical values, the widget applies heuristics to determine other types of visual encodings. For example, when connecting a set of quantitative values to one axis and a set of categorical values to the other, the widget generates a bar chart. The widget therefore decides on the marks that compose the visualization, i.e., the data input and the order in which it is connected determines the type and layout of the resulting visualization. This idea is comparable to Tableau's "Columns and Rows" system where marks and visual properties are determined by the data attributes dropped onto each shelf.

\section{Simple Representations as Building Blocks}

$\mathrm{P} 1$ proposed an idea where the tool determines the visualization design based on the involved data attribute types. For example, Figure 10a (top row) shows two visualizations automatically generated by the tool for two data attributes: "[the tool] knows it [the city attribute] is categorical, so it makes up something like this. For the salary, it creates a distribution [histogram] of the actual values themselves." [P1]. To define more complex visualizations, the user can merge simple representations via drag-and-drop gestures (see Fig. 10b, bottom, showing "the average salary of every city" [P1]). In this solution, all visualization design decisions are made by the tool based on the data attributes. The more attributes involved, the more complex the decisions inferred by the tool. Although not sketched, P1 mentioned that "there will be ways to sort of customize this [resulting visualization], 'cause you are gonna want to customize them in different ways, presumably. But it [the tool] will give you something for a start. It will give you something basic to work with and then you can carry that on."

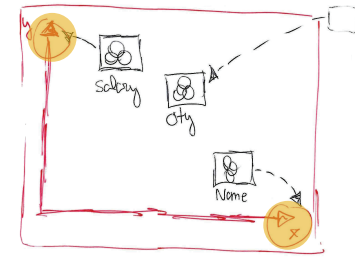

(a) P3.

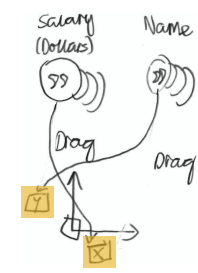

(b) P4.

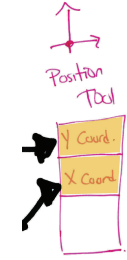

(c) P12.
Figure 9: Axis-based solutions. The yellow highlighting indicates the axes' points where data can be connected.
Chart Galleries

In their designs P3, 4 and 13 proposed a gallery of charts for the user to select in order to represent data attributes (Fig. 11). P13 explained that this part of his design was similar to MS Excel's charts gallery or Tableau's "Show Me" pane. The galleries by P3 and P4 additionally provide access to other type of designs. For example, dragging a bar chart from P3's gallery onto the canvas creates an axis-based widget.

\section{FAMILIES OF DESIGN SOLUTIONS}

Our participants' design ideas presented above and our own in-house solutions can be grouped into three vis tool design families: visualization by example, collective proxy objects, and automated principled design (see Fig. 12). We characterize these below alongside their underlying design strategies: automated iteration, abstraction, and automated choices.

\section{Visualization-by-Example: Automated Iteration}

Four of the presented design ideas require the manual specification of visual mappings based on a single data instance (e.g., one table row) which are then automatically applied to other data or the entire set (Fig. 12, left). The P6's "plus button" (Fig. 6) and P10's gesture-based mapping propagation idea represent this visualization-by-example approach, as well as P13's, our own description of a Macro Recorder (Fig. 1), and comparable solutions in commercial tools such as MS Excel.

These solutions directly address the issue of having to repeatedly specify the mapping between individual data points and visual elements as common in bottom-up visualization tools. Visualization-by-example solutions implement automated iteration as a strategy that allows the automatic application of design decisions reflected by an individual data-driven visual element to a larger scale. As shown above, implementations of this strategy differ in terms of how automated iterations actions are specified and when results become visible. For example, P6's "plus button" idea specifies how a data instance is represented by a mark's visual properties. In contrast, macro

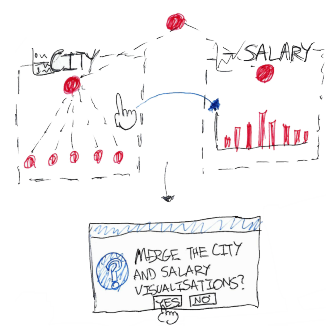

(a) Individual views.

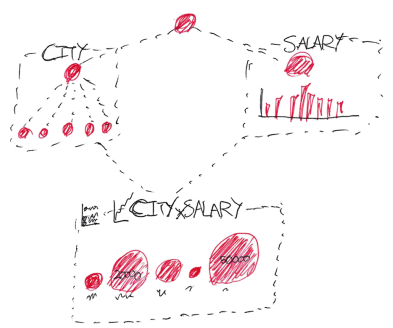

(b) Resulting Visualization.
Figure 10: Merging simple views to create more complex one [P1]. 


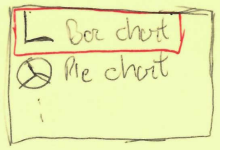

(a) P3.

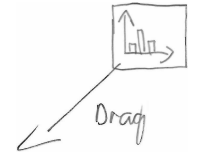

(b) P4.

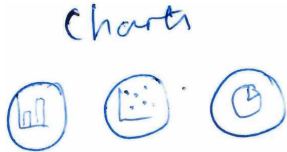

(c) P13.
Figure 11: Sketched chart galleries.

recorder solutions specify mappings as ordered sequences of actions carried out within a particular temporal interval. Both solutions apply mappings in bulk, while others, such as P10's gesture-based mapping propagation, do this as new individual mappings steps are specified, showing how the automated iteration is progressively carried out across the data.

\section{Collective Proxy Objects: Abstraction}

Eight sketches implement interface elements (e.g., a widget), separate from the resulting visualization, to specify visual mappings between data and visual elements (Fig. 12, middle). Such proxy objects can take connections carrying individual values, sets, or data attributes. Modifications to the visual mapping happen through the proxy object and are propagated to all the corresponding visualization elements. The design solutions in Figures 3, 7b, and 8 show different implementations of collective proxy objects. Other examples include Tableau's "Marks Card". P2's presents a different implementation of this design approach in which every visualization element is also a proxy for all other elements within its group (Fig. 7a).

Design solutions in this family are based on abstraction as a design strategy: a single interface element represents multiple data items and the visual properties of corresponding marks while the individual constituents are hidden. Abstraction as a general term is well discussed in psychology, semiotics and other areas, but here it best conveys this strategy's meaning. As a design strategy to scale constructive visualizations, abstraction affects access to visual properties and mapping options, and to the data itself: a high degree of abstraction may allow visual mappings at an attribute-level, while a low degree will enable mappings at the level of individual data values.

\section{Automated Principled Design: Automated Choices}

Seven of the presented designs support a top-down approach to visualization construction [44], where visual mappings are determined and automatically applied by the visualization tool based on pre-defined principles as specified in its implementation (Fig. 12, right). For example, in P4's solution (Fig. 9b) the designer selects the data attributes and loosely specifies the visualization type by mapping each to the chart's axes. The tool then determines the chart type that fits these data types. Similarly, in P1's design, two visualizations are automatically merged into one, based on the initial data types involved (Fig. 10). In P13's solution, the tool offers a variety of chart types which will fit the attribute types selected by the

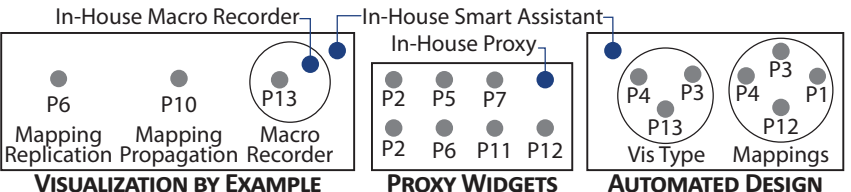

Figure 12: Families of visual mapping strategies. Gray circles represent the participants' designs; in-house solutions appear in blue. designer. Once the designer selects the chart type, the tool applies the necessary visual mappings. This is similar to how MS Excel or Tableau's "Show Me" pane operate. P3's solution (Fig. 9a) supports scenarios where the designer chooses a visualization type (e.g., a bar chart), but then maps data attributes of an unsuitable type (e.g., two continuous attributes): the tool automatically performs operations to make the data fit the visualization type (e.g., bin or average data values).

The underlying strategy of this family of designs is that of automated choices where explicit decisions by the designer are minimized, and the tool makes choices on: (1) the visualization type (e.g., the type of marks and visual attributes), (2) the mappings between data and visual variables, (3) the data or data subset to which to apply mappings (e.g., eliminating outliers), and (4) the data transformations (e.g., binning a continuous data attribute to map to a discrete visual variable). While this strategy leaves many design decisions to the tool, automated choices can be implemented as mere suggestions or as configurable by the designer.

The three strategies to scale constructive visualization approaches to large datasets-automated iteration, abstraction, and automated choices - are not mutually exclusive, but can be combined. For example, our "Smart Assistant" solution (Fig. 2) can be considered as an implementation of the automated choices strategy, as it makes unprompted decisions about generalizing visual mappings, but it also implements the automated iteration strategy as it allows the designer to explicitly apply visual mappings to additional data points.

\section{AGENCY AND DATA GRANULARITY: A DESIGN SPACE}

The families described above are clusters of design solutions based on strategies to implement constructive visualization tools that scale to larger datasets. In this section we present a design space emerging from these strategies which is defined by two qualitative dimensions: agency and granularity. This design space summarizes the differences between the proposed solutions also in relation to previous research and existing commercial visualization tools.

\section{Agency}

Agency refers to who carries out the visualization process: the tool or the human designer. A visualization tool can make a variety of decisions throughout the design process, such as how to transform a continuous data value to map it to a discrete visual variable ([P3]), selecting the visualization type suitable for a designer-selected data subset (Fig. 11c [P13]), or even performing actions on behalf of the designer, such as applying a mapping repetitively to the full dataset (Fig. 9b [P4]) or just to the subsequent record (Fig. 6 [P6]).

This dimension is represented as the horizontal axis in the design space diagram shown in Figure 13. Design solutions where more agency is placed on the visualization tool, that is, where the tool is in charge of important mapping decisions and actions, are located more to the right. To the left on this axis are purely constructive tools, such as iVoLVER. ${ }^{3}$

\footnotetext{
${ }^{3}$ Note that even a tool like iVoLVER, built from the ground up with a constructive philosophy, makes some decisions for the designer. For example, if the designer drops a data value on top of a mark, iVoLVER will automatically assign it to a default visual property.
} 
Agency is not exclusively linked to the automatic choices strategy. Abstraction and automated iteration also place some agency on the tool, as it takes over designer activities.

\section{Granularity}

Granularity refers to the level at which the tool allows the manipulation of both data and visual representations, that is, what designers can see and manipulate. At the level of finegranularity the tool provides access to individual data values (e.g., a single number in a table cell) and allows for the manipulation of individual marks (e.g., a single bar in a bar chart). This is how iVoLVER operates. Tools with a coarse granularity operate on groups of values, at an attribute level, or on groups of attributes (or on groups of marks, or even groups of small multiples on the visual side). For example, the solutions of the collective proxy objects family specify the visual mappings of many marks through a single widget that takes data attributes-not individual values-(e.g., Fig. 8a [P2]).

This dimension is represented as the vertical axis in the design space diagram of Figure 13. Solutions lower in this axis have a fine granularity (e.g., iVoLVER, which is based on the manipulation of individual values and marks); solutions higher up represent data mostly at an attribute level (e.g, the data column becomes a single element to manipulate in Tableau, ExPlates [35], Many Eyes [70], and the designs of Fig. 8). In DataMeadow [14], the visualization process involves manipulating data attributes, but the entire dataset can also be represented and manipulated through a single visual element. Lark [68] supports an even more coarse-grained approach: a complete dataset is represented as a single visual element that moves through different transformation steps and the visualization's components cannot be manipulated directly.

\section{The Design Space: Strategies, Quadrants \& Limitations}

The set of strategies described in the Families of Design Solutions section can also be described as a collection of design tropes that allow visualization tool designers to explore and discuss important options and their corresponding consequences. For example, adding automatic iteration to a purely constructive tool will place more agency on the tool: now the tool does something for the designer. Adding abstraction, decreases the level of granularity (abstraction hides access to the more atomic elements) and also places agency on the tool: as with automatic iteration the tool applies operations to all individual data values and visuals on behalf of the designer. Finally, implementing automated choices shifts the agency balance to the tool side; adding automated decisions to any tool will move it closer to the right end of the design space.

Besides the in-house (red) and workshop (blue) design solutions, we have placed a selection of representative existing visualization tools (green) into this design space defined by four quadrants Q1-Q4 (see Fig. 13). While not exhaustive in terms of included tools, the resulting distribution leads to interesting observations. First, most included tools (green) gravitate towards the upper part of the space (Q3 and Q4). Second, the right side of Q1 is populated mostly by solutions from the workshop (red). Third, Q2, which represents tools that make many decisions on behalf of the designer but stay at a coarse-grained granularity level, is barely populated. We

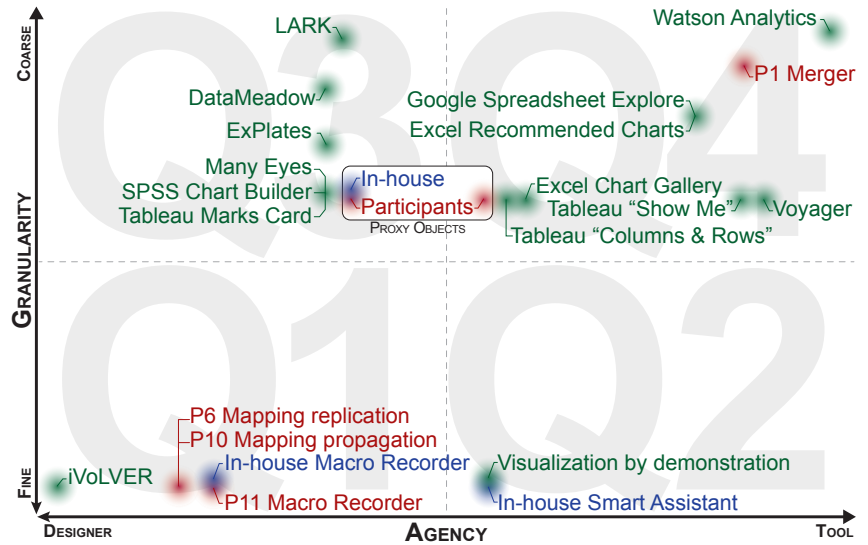

Figure 13: Design space defined by agency \& granularity with existing tools (green), including MS Excel [15], the Google Sheets Explorer feature [17], and SPSS Chart Builder [33], alongside in-house (blue) and workshop solutions (red).

believe that there is potential for tools in this category. For example, a Q2 tool could be a "visualization tutor" that walks a learner step-by-step through a visual mapping process based on individual data values.

The design space should be understood as a qualitative tool as opposed to an exact quantitative metric to specify tool differences. Therefore the positioning of tools and solutions in Figure 13 is open to interpretation and is meant to promote discussion. Some tools also appear in several places because they incorporate multiple approaches to creating visualizations (e.g., Tableau's "Show Me" pane and "Columns and Rows"). Similarly, the workshop solutions that we grouped as "collective proxy objects" span horizontally because they combine abstraction at different levels of automated choices.

\section{DISCUSSION}

In this section we interpret our results, explain the relevance of our work, discuss its implications, and provide a critical look at the current landscape of visualization tools.

\section{The Agency-Granularity Design Space}

Our design explorations generate a design space based on agency and granularity that conceptualizes the challenge of scale in visualization tools. We see this design space as a lens to look at and discuss existing design solutions and strategies and to inspire new ones. It brings constructive approaches into view in relation to more conventional tools, offering designers a catalog of examples and a deeper understanding of available solutions. It might also help the visualization community to identify promising unexplored spaces which we have started to highlight. We believe that this space complements and further refines other characterizations of the existing landscape of visualization tools, such as Tory and Möller's taxonomy [69] and Méndez et al.'s top-down vs. bottom-up continuum [44].

\section{Building Better Visualization Tools}

The overarching goal of our work is to help create better visualization tools. This means designing tools that can deal with datasets that are larger than just a few records (scalability), that are easy to use but also inspire critical exploration, that are transparent and understandable, and that support mindful design, as well as ownership of the results by the designer. 
The strategies presented here help deal with the scalability issue, but not all of them address the other desirable outcomes listed above. Previous research indicates that tools located far from the bottom-left corner of the design space, while increasing scalability, can have a negative impact on other desirable characteristics [44]. The next step is therefore to find the "sweet spot" that maximizes the advantages. Our work supports this goal but further research is needed to investigate how different levels of agency and granularity affect the use of visualization tools. Moreover, where the sweet spot is will depend on the particular task at hand (e.g., new vs. familiar data), the audience (e.g., expertise), and the usage scenario.

We suspect that, from the strategies described as part of the families of design solutions, automated iteration could retain most of the advantages of constructive tools while addressing the scalability problem. A possible alternative is to design tools that adapt their interfaces to support different stages of the design process and expertise depending on the circumstances. To a small extent some tools such as MS Excel and Tableau already address different levels of agency and granularity, but we believe there is much space for improvement.

\section{A Critical Look at the Current Landscape of Vis Tools}

Placing a selection of existing visualization tools into our design space defined by agency and granularity (see Fig. 13) suggests that much effort has gone into creating tools that support the rapid design of visualizations through coarse data granularity and, arguably, little effort from the designer (see Q3 and Q4). From our own experience, such tools are highly valuable, and we use them ourselves as part of our design, research, and teaching practice. However, while supporting rapid visualization creation in this way (through pre-defined or AI-inferred design choices) has its place, we argue that there is a need for more alternatives. Recent work on supporting more manual constructive visualization processes [30, 45] and physicalization $[29,31]$ is already going in this direction, but there is an urgency for more approaches like this. Data analysis and required literacy [9] is starting to have an effect beyond professional scenarios and on our personal lives [26] including even the youngest members of society [3, 42].

A visualization is not just a means to an end. Reflection on the data and insight generation take place also during the process of constructing the visualization [23, 71]. Supporting a fast visualization process is therefore not necessarily more "efficient", as it may gloss over important details of the data, patterns, uncertainties, open questions, and opportunities for exploration and critique. We know from (data) design practice that active involvement [49], and repetition [5] can foster creativity and critical thinking. When we consider visualization as an active data exploration process, the visualization tool itself might heavily influence insights and reflections. But even if we consider visualization as something to be consumed rather than actively explored and manipulated in a hands-on way, the tool plays an important role. There is a rhetorical nature to visualizations [27], where choices in visual representation and composition steer our thinking of the presented topics. Visualization tools influence the design process of visualizations and, through their implementations, can make decisions on behalf of the designer. Their power to influence messages "by design" should not be underestimated. As visualization tool designers we have a responsibility, and we hope that the design space presented in this paper will fuel a discussion in the community and inspire new and alternative avenues to visualization tool design. In fact, linking our findings to philosophy and cognitive science, cognitive embodiment [11], media studies [41], feminist theory [66] and, most relevantly, the study of agency and technology $[38,66]$ may lead to even more research avenues.

\section{LIMITATIONS AND QUESTIONS FOR FUTURE WORK}

Although our conceptualization of the problem, the catalog of examples and strategies, and the proposed design space advance our understanding in the area, there is much left to do, especially characterizing the effects of the different design strategies on the outcomes that we care about. Perhaps most interesting for future research is to investigate how different design strategies impact the visualization design process, in particular, regarding mindful and critical design, transparency, and authorship.

We also highlight that the outcomes of our design workshops should not be interpreted as quantitative evidence of what strategy or type of tool is best understood or comes most "naturally" to participants. Our workshops were designed to find as many divergent solutions as possible, that is, to expose the nature of the design space, rather than identifying a "best" design. They were not meant to serve as a faithful characterization of what people desire or understand. Although we explicitly set out to include people with different backgrounds and levels of expertise, our sampling is small and not fully representative of all potential audiences. Additional studies are needed to investigate approaches of other populations (e.g., professionals). Our workshops are also limited in other ways: participants are influenced (1) by the tools they know, and (2) by the tools we used in our study to contextualize the problem; and (3) they might need more time to provide a wider variety of designs.

\section{CONCLUSION}

We have explored the design space of visualization tools that could combine the benefits of constructive, "bottom-up" visualization tools, namely transparency and active involvement in the visualization process, with those of "top-down" approaches, such as rapid visualization creation and applicability to large datasets. We analyzed solutions proposed by ourselves and a group of participants with a range of skill sets and levels of expertise in visualization. From this data we derived a catalog of solutions, a set of three design strategies (automated iteration, abstraction, and automated choices) and propose a conceptualization of the design space of visualization tools based on agency and granularity. Finally, based on our analysis we provide a critical discussion of the existing landscape of tools, which we hope will help designers and the community consider better options in the design of visualization tools.

\section{ACKNOWLEDGMENTS}

We thank all our study participants for taking part in our design workshops. The Ecuadorian Government supports Gonzalo Gabriel Méndez through a SENESCYT scholarship. 


\section{REFERENCES}

1. Edith Ackermann. 2001. Piaget's constructivism, Papert's constructionism: What's the difference. Future of learning group publication 5, 3 (2001), 438.

2. James F. Allen, Curry I. Guinn, and Eric Horvitz. 1999. Mixed-initiative interaction. IEEE Intelligent Systems 14, 5 (1999), 14-23. DOI :

http://dx.doi.org/10.1109/5254.796083

3. Basak Alper, Nathalie Henry Riche, Fanny Chevalier, Jeremy Boy, and Metin Sezgin. 2017. Visualization Literacy at Elementary School. In Proceedings of the 2017 CHI Conference on Human Factors in Computing Systems (CHI '17). ACM, New York, NY, USA, 5485-5497. DOI :

http://dx.doi.org/10.1145/3025453.3025877

4. Daniel Archambault, Tamara Munzner, and David Auber. 2008. GrouseFlocks: Steerable Exploration of Graph Hierarchy Space. IEEE Transactions on Visualization and Computer Graphics 14, 4 (July 2008), 900-913. DOI : http://dx.doi. org/10.1109/TVCG. 2008.34

5. Alex Bigelow, Steven Drucker, Danyel Fisher, and Miriah Meyer. 2014. Reflections on How Designers Design with Data. In Proceedings of the 2014 International Working Conference on Advanced Visual Interfaces (AVI '14). ACM, New York, NY, USA, 17-24. DOI : http://dx.doi.org/10.1145/2598153.2598175

6. Fearn Bishop and Uta Hinrichs. 2017. Challenges of Running Constructive Visualization Studies with Children. In DIS'17 workshop on Pedagogy \& Physicalization: Designing Learning Activities around Physical Data Representations. http://dataphys.org/workshops/dis17

7. Michael Bostock, Vadim Ogievetsky, and Jeffrey Heer. 2011. D3; Data-Driven Documents. IEEE Transactions on Visualization and Computer Graphics 17, 12 (Dec. 2011), 2301-2309. DOI :

http://dx.doi.org/10.1109/TVCG.2011.185

8. Fatma Bouali, Abdelheq Guettala, and Gilles Venturini. 2016. VizAssist: an interactive user assistant for visual data mining. The Visual Computer 32, 11 (01 Nov 2016), 1447-1463. DOI :

http://dx.doi.org/10.1007/s00371-015-1132-9

9. Jeremy Boy, Ronald A. Rensink, Enrico Bertini, and Jean-Daniel Fekete. 2014. A Principled Way of Assessing Visualization Literacy. IEEE Transactions on Visualization and Computer Graphics 20, 12 (Dec 2014), 1963-1972. DOI :

http://dx.doi.org/10.1109/TVCG.2014.2346984

10. Liz Carver and Murray Turoff. 2007. Human-computer Interaction: The Human and Computer As a Team in Emergency Management Information Systems. Commun. ACM 50, 3 (March 2007), 33-38. DOI : http://dx.doi.org/10.1145/1226736.1226761
11. Andy Clark and David Chalmers. 1998. The Extended Mind. Analysis 58, 1 (1998), 7-19. http://www. jstor.org/stable/3328150

12. David Coyle, James Moore, Per Ola Kristensson, Paul Fletcher, and Alan Blackwell. 2012. I Did That! Measuring Users' Experience of Agency in Their Own Actions. In Proceedings of the SIGCHI Conference on Human Factors in Computing Systems (CHI '12). ACM, New York, NY, USA, 2025-2034. DOI : http://dx.doi.org/10.1145/2207676.2208350

13. Frédéric Dehais, Mickaël Causse, François Vachon, and Sébastien Tremblay. 2012. Cognitive conflict in human-automation interactions: a psychophysiological study. Applied ergonomics 43, 3 (2012), 588-595. DOI: http://dx.doi.org/10.1016/j. apergo. 2011.09.004

14. Niklas Elmqvist, John Stasko, and Philippas Tsigas. 2008. DataMeadow: A Visual Canvas for Analysis of Large-scale Multivariate Data. Information Visualization 7, 1 (March 2008), 18-33. DOI : http://dx.doi.org/10.1145/1391107.1391110

15. Microsoft Excel. 2017. Create a chart with recommended charts - Excel. (2017).

https://support.office.com/en-gb/article/ Create-a-chart-with-recommended-charts-cd131b77-79c7 -4537-a438-8db20cea84c0 [Online; accessed 16-September-2017].

16. Sara Carolyn Fisher. 1916. The process of generalizing abstraction; and its product, the general concept. The Psychological Monographs 21, 2 (1916), i-218. DOI: http://dx.doi.org/10.1037/h0093097

17. Google. 2017. See and use suggested charts and analysis in a spreadsheet. (2017).

https://support. google.com/docs/answer/6280499 [Online; accessed 16-September-2017].

18. David Gotz and Zhen Wen. 2009. Behavior-driven Visualization Recommendation. In Proceedings of the 14th International Conference on Intelligent User Interfaces (IUI '09). ACM, New York, NY, USA, 315-324. DOI : http://dx.doi.org/10.1145/1502650.1502695

19. Lars Grammel, Chris Bennett, Melanie Tory, and Margaret-Anne Storey. 2013. A Survey of Visualization Construction User Interfaces. In EuroVis - Short Papers, Mario Hlawitschka and Tino Weinkauf (Eds.). The Eurographics Association. DOI : http://dx.doi.org/10. 2312/PE. EuroVisShort. EuroVisShort2013.019-023

20. Patrick Haggard and Manos Tsakiris. 2009. The Experience of Agency. Current Directions in Psychological Science 18, 4 (2009), 242-246. DOI : http://dx.doi.org/10.1111/j.1467-8721.2009.01644.x

21. Orit Hazzan. 2003. How Students Attempt to Reduce Abstraction in the Learning of Mathematics and in the Learning of Computer Science. Computer Science Education 13, 2 (2003), 95-122. DOI: http://dx.doi.org/10.1076/csed.13.2.95.14202 
22. Christian Heath, Jon Hindmarsh, and Paul Luff. 2010. Video in Qualitative Research. SAGE Publications. https://books.google.co.uk/books?id=MtmViguNi4UC

23. Uta Hinrichs and Stefania Forlini. 2017. In defense of sandcastles: research thinking through visualization in DH. In Proceedings of the conference on Digital Humanities. International Alliance of Digital Humanities Organizations (ADHO).

https://dh2017. adho.org/abstracts/133/133.pdf

24. Douglas Hofstadter and Emmanuel Sander. 2013. Surfaces and Essences: Analogy as the Fuel and Fire of Thinking. Basic Books.

https://books.google.ca/books?id=XkQT5eTnurYC

25. Eugene Robert Hoyt, Dallas Snider, Carla Thompson, and Sarita Mantravadi. 2016. IBM Watson Analytics: Automating Visualization, Descriptive, and Predictive Statistics. JMIR Public Health Surveill 2, 2 (11 Oct 2016), e157. DOI : http://dx.doi.org/10.2196/publichealth.5810

26. Dandan Huang, Melanie Tory, Bon Adriel Aseniero, Lyn Bartram, Scott Bateman, Sheelagh Carpendale, Anthony Tang, and Robert Woodbury. 2015. Personal Visualization and Personal Visual Analytics. Visualization and Computer Graphics, IEEE Transactions on 21, 3 (March 2015), 420-433. DOI :

http://dx.doi.org/10.1109/TVCG. 2014.2359887

27. Jessica Hullman and Nick Diakopoulos. 2011. Visualization Rhetoric: Framing Effects in Narrative Visualization. IEEE Transactions on Visualization and Computer Graphics 17, 12 (Dec 2011), 2231-2240. DOI : http://dx.doi.org/10.1109/TVCG.2011.255

28. Samuel Huron. 2014. Constructive Visualization : A token-based paradigm allowing to assemble dynamic visual representation for non-experts. Theses. Université Paris Sud - Paris XI.

https://tel.archives-ouvertes.fr/tel-01126892

29. Samuel Huron, Sheelagh Carpendale, Jeremy Boy, and Jean-Daniel Fekete. 2016. Using VisKit: A Manual for Running a Constructive Visualization Workshop. In Pedagogy of Data Visualization Workshop at IEEE VIS 2016. Baltimore, MD, United States.

https://hal.inria.fr/hal-01384388

30. Samuel Huron, Sheelagh Carpendale, Alice Thudt, Anthony Tang, and Michael Mauerer. 2014. Constructive Visualization. In Proceedings of the 2014 Conference on Designing Interactive Systems (DIS '14). ACM, New York, NY, USA, 433-442. DOI : http://dx.doi.org/10.1145/2598510.2598566

31. Samuel Huron, Pauline Gourlet, Uta Hinrichs, Trevor Hogan, and Yvonne Jansen. 2017. Let's Get Physical: Promoting Data Physicalization in Workshop Formats. In Proceedings of the 2017 Conference on Designing Interactive Systems (DIS '17). ACM, New York, NY, USA, 1409-1422. DOI :

http://dx.doi.org/10.1145/3064663.3064798
32. Samuel Huron, Yvonne Jansen, and Sheelagh Carpendale. 2014. Constructing Visual Representations: Investigating the Use of Tangible Tokens. Visualization and Computer Graphics, IEEE Transactions on 20, 12 (Dec 2014), 2102-2111. DOI :

http://dx.doi.org/10.1109/TVCG.2014.2346292

33. IBM. 2017. IBM Knowledge Center - Chart Builder layout and terms. (2017).

https://www. ibm.com/support/knowledgecenter/en/SSLVMB_20. $0.0 /$ com.ibm.spss.statistics.help/chart_create_layout.htm [Online; accessed 16-September-2017].

34. Waqas Javed. and Niklas Elmqvist. 2013. ExPlates: Spatializing Interactive Analysis to Scaffold Visual Exploration. Computer Graphics Forum 32, 3pt4 (2013), 441-450. DOI : http://dx.doi.org/10.1111/cgf.12131

35. Waqas Javed and Niklas Elmqvist. 2013. ExPlates: Spatializing Interactive Analysis to Scaffold Visual Exploration. Computer Graphics Forum 32, 3pt4 (2013), 441-450. DOI : http://dx.doi.org/10.1111/cgf.12131

36. Gregor Kiczales. 1996. Beyond the black box: open implementation. IEEE Software 13, 1 (Jan 1996), 8, 10-11. DOI : http://dx.doi.org/10.1109/52.476280

37. Philipp Koytek, Charles Perin, Jo Vermeulen, Elisabeth André, and Sheelagh Carpendale. 2017. MyBrush: Brushing and Linking with Personal Agency. IEEE Transactions on Visualization and Computer Graphics PP, 99 (2017), 1-1. DOI :

http://dx.doi.org/10.1109/TVCG.2017.2743859

38. Bruno Latour and Couze Venn. 2002. Morality and Technology. Theory, Culture \& Society 19, 5-6 (2002), 247-260. DOI :

http://dx.doi.org/10.1177/026327602761899246

39. Shixia Liu, Weiwei Cui, Yingcai Wu, and Mengchen Liu. 2014. A survey on information visualization: recent advances and challenges. The Visual Computer 30, 12 (01 Dec 2014), 1373-1393. DOI :

http://dx.doi.org/10.1007/s00371-013-0892-3

40. Jock Mackinlay, Pat Hanrahan, and Chris Stolte. 2007. Show Me: Automatic Presentation for Visual Analysis. IEEE Transactions on Visualization and Computer Graphics 13, 6 (Nov 2007), 1137-1144. DOI : http://dx.doi.org/10.1109/TVCG.2007.70594

41. Marshall McLuhan. 2002. Understanding Media: The Extensions of Man (critical edition ed.). Gingko Press, Inc, Corte Madera, CA.

42. Emily McReynolds, Sarah Hubbard, Timothy Lau, Aditya Saraf, Maya Cakmak, and Franziska Roesner. 2017. Toys That Listen: A Study of Parents, Children, and Internet-Connected Toys. In Proceedings of the 2017 CHI Conference on Human Factors in Computing Systems (CHI '17). ACM, New York, NY, USA, 5197-5207. DOI : http://dx.doi.org/10.1145/3025453.3025735 
43. Honghui Mei, Yuxin Ma, Yating Wei, and Wei Chen. 2017. The design space of construction tools for information visualization: A survey. Journal of Visual Languages \& Computing (2017). DOI : http://dx.doi.org/10.1016/j.jvlc. 2017.10.001

44. Gonzalo Gabriel Méndez, Uta Hinrichs, and Miguel A. Nacenta. 2017. Bottom-up vs. top-down: trade-offs in efficiency, understanding, freedom and creativity with InfoVis tools. In Proceedings of the 2017 CHI Conference on Human Factors in Computing Systems (CHI '17). ACM, New York, NY, USA, 13. DOI : http://dx.doi.org/10.1145/3025453.3025942

45. Gonzalo Gabriel Méndez, Miguel A. Nacenta, and Sebastien Vandenheste. 2016. iVoLVER: Interactive Visual Language for Visualization Extraction and Reconstruction. In Proceedings of the 2016 CHI Conference on Human Factors in Computing Systems (CHI '16). ACM, New York, NY, USA, 4073-4085. DOI : http://dx.doi.org/10.1145/2858036.2858435

46. Thomas Mühlbacher, Harald Piringer, Samuel Gratzl, Michael Sedlmair, and Marc Streit. 2014. Opening the Black Box: Strategies for Increased User Involvement in Existing Algorithm Implementations. IEEE Transactions on Visualization and Computer Graphics 20, 12 (Dec 2014), 1643-1652. DOI :

http://dx.doi.org/10.1109/TVCG.2014.2346578

47. Jurriaan D Mulder, Jarke J Van Wijk, and Robert Van Liere. 1999. A survey of computational steering environments. Future generation computer systems 15, 1 (1999), 119-129. DOI : http://dx.doi.org/10.1016/s0167-739x(98)00047-8

48. Brad A. Myers. 1992. Demonstrational Interfaces: A Step Beyond Direct Manipulation. Computer 25, 8 (1992), 61-73. DOI : http://dx.doi.org/10.1109/2.153286

49. Bettina Nissen and John Bowers. 2015. Data-Things: Digital Fabrication Situated Within Participatory Data Translation Activities. In Proceedings of the 33rd Annual ACM Conference on Human Factors in Computing Systems (CHI'15). ACM, New York, NY, USA, 2467-2476. DOI : http://dx.doi.org/10.1145/2702123.2702245

50. Kostas Pantazos and Søren Lauesen. 2012. Constructing Visualizations with InfoVis Tools - An Evaluation from a user Perspective. GRAPP/IVAPP 2012 (2012), 731-736. http://www.it-c.dk/people/slauesen/S-EHR/Constructing_ Visualizations.pdf

51. Seymour Papert. 1993. Mindstorms: Children, Computers, and Powerful Ideas. Basic Books, New York.

52. Seymour Papert and Idit Harel. 1991. Situating Constructionism. In Constructionism. Ablex Publishing Corporation.

53. Raja Parasuraman and Dietrich H. Manzey. 2010. Complacency and Bias in Human Use of Automation: An Attentional Integration. Human Factors 52, 3 (2010), 381-410. DOI :

http://dx.doi.org/10.1177/0018720810376055
54. Raja Parasuraman, Robert Molloy, and Indramani L Singh. 1993. Performance Consequences of Automation-Induced 'Complacency'. The International Journal of Aviation Psychology 3, 1 (1993), 1-23. DOI : http://dx.doi.org/10.1207/s15327108ijap0301_1

55. Charles Perin, Pierre Dragicevic, and Jean-Daniel Fekete. 2014. Revisiting Bertin Matrices: New Interactions for Crafting Tabular Visualizations. IEEE Transactions on Visualization and Computer Graphics 20, 12 (Dec 2014), 2082-2091. DOI :

http://dx.doi.org/10.1109/TVCG. 2014.2346279

56. Daniel B. Perry, Bill Howe, Alicia M.F. Key, and Cecilia Aragon. 2013. VizDeck: Streamlining exploratory visual analytics of scientific data (iConference 2013). iSchools. http://hdl. handle. net/2142/36044

57. Willard Van Orman Quine. 2016. Levels of Abstraction (1972). Palgrave Macmillan UK, London, 12-20. DOI : http://dx.doi.org/10.1057/9781137472519_3

58. Bahador Saket, Hannah Kim, Eli T. Brown, and Alex Endert. 2017. Visualization by Demonstration: An Interaction Paradigm for Visual Data Exploration. IEEE Transactions on Visualization and Computer Graphics 23, 1 (Jan 2017), 331-340. DOI :

http://dx.doi.org/10.1109/TVCG. 2016.2598839

59. Ben Schneiderman and Catherine Plaisant. 1998. Designing the user interface. (1998).

60. Burrhus Frederic Skinner. 1977. Why I Am Not a Cognitive Psychologist. Behaviorism 5, 2 (1977), 1-10. http://www. jstor.org/stable/27758892

61. Alan Snyder. 1986. Encapsulation and Inheritance in Object-oriented Programming Languages. In Conference Proceedings on Object-oriented Programming Systems, Languages and Applications (OOPSLA '86). ACM, New York, NY, USA, 38-45. DOI : http://dx.doi.org/10.1145/28697.28702

62. Tableau Software. 2017a. Business Intelligence and Analytics. (2017). https://www. tableau.com

63. Tableau Software. 2017b. Columns and Rows Shelves. (2017). http://onlinehelp. tableau.com/current/pro/ desktop/en-us/buildmanual_shelves_columnrow.html [Online; accessed 19-September-2017].

64. Tableau Software. 2017c. Data Pane. (2017). http://onlinehelp. tableau.com/current/pro/desktop/en-us/ environ_workspace_datawindow.html [Online; accessed 19-September-2017].

65. Tableau Software. 2017d. Marks Card. (2017). http://onlinehelp. tableau.com/current/pro/desktop/en-us/ buildmanual_shelves_marks.html [Online; accessed 19-September-2017].

66. Lucy Suchman. 2007. Agencies in technology design: Feminist reconfigurations. (2007).

http://citeseerx.ist.psu . edu/viewdoc/download?doi=10.1.1. 548.7968\&rep=rep $1 \&$ type=pdf 
67. Guo-Dao Sun, Ying-Cai Wu, Rong-Hua Liang, and Shi-Xia Liu. 2013. A Survey of Visual Analytics Techniques and Applications: State-of-the-Art Research and Future Challenges. Journal of Computer Science and Technology 28, 5 (Sept. 2013), 852-867. DOI :

http://dx.doi.org/10.1007/s11390-013-1383-8

68. Matthew Tobiasz, Petra Isenberg, and Sheelagh Carpendale. 2009. Lark: Coordinating Co-located Collaboration with Information Visualization. IEEE Transactions on Visualization and Computer Graphics 15, 6 (Nov. 2009), 1065-1072. DOI :

http://dx.doi.org/10.1109/TVCG.2009.162

69. Melanie Tory and Torsten Möller. 2004. Rethinking Visualization: A High-Level Taxonomy. In IEEE Symposium on Information Visualization. 151-158. DOI : http://dx.doi.org/10.1109/INFVIS.2004.59

70. Fernanda B. Viegas, Martin Wattenberg, Frank van Ham, Jesse Kriss, and Matt McKeon. 2007. ManyEyes: a Site for Visualization at Internet Scale. IEEE Transactions on
Visualization and Computer Graphics 13, 6 (Nov. 2007), 1121-1128. DOI :

http://dx.doi.org/10.1109/TVCG.2007.70577

71. Jagoda Walny, Samuel Huron, and Sheelagh Carpendale. 2015. An Exploratory Study of Data Sketching for Visual Representation. Computer Graphics Forum 34, 3 (2015), 231-240. DOI : http://dx.doi.org/10.1111/cgf.12635

72. Kanit Wongsuphasawat, Dominik Moritz, Anushka Anand, Jock Mackinlay, Bill Howe, and Jeffrey Heer. 2016. Voyager: Exploratory Analysis via Faceted Browsing of Visualization Recommendations. IEEE Transactions on Visualization and Computer Graphics 22, 1 (Jan 2016), 649-658. DOI :

http://dx.doi.org/10.1109/TVCG.2015.2467191

73. Guo Yu and Alan Blackwell. 2017. Effects of Timing on Users' Agency during Mixed-Initiative Interaction. In Proceedings of the 2017 British Human Computer Interaction Conference (BHCI'17).

http://hci2017.bcs.org/wp-content/uploads/21.pdf 